\title{
AS-mixed Network Models Created By Triangle-expanding Operations
}

\author{
Jing $\mathrm{Su}^{1,}$, , Fei $\mathrm{Ma}^{2}$, a, Bing $\mathrm{Yao}^{3,}$ a ${ }^{*}$, Ming $\mathrm{Yao}^{4, ~ b}$ \\ ${ }^{a}$ College of Mathematics and Statistics, Northwest Normal University, \\ Lanzhou, Gansu CHINA 730070 \\ bDepartment of Information Processand Control Engineering, Lanzhou \\ Petrochemical College of Vocational Technology \\ Lanzhou, Gansu CHINA 730070 \\ *yybb@163.com
}

Keywords: Network model; Operations; Apollonian networks; Degree cumulative distribution; Velocity.

\begin{abstract}
We build up network operations for constructing large scale of dynamic networks from the known network models having high quality and good topological property. In this paper, we defined triangle-expanding operation and triangle-contracting operation, as well as construct the new random AS-mixed network models on the basis of Apollonian network models by using these two kinds of operations. We prove the scale-free of new network models and explain the closeness of operations to desired network properties.
\end{abstract}

\section{Introduction}

In the recent years we have witnessed a spring tide in the research of a wide range of complex systems which can be described in terms of networks, we can explore the nature of complex systems by researching the structure of network models. We know most of the real networks are random, So, a variety of network models rised in response to the proper moment (Ref. [1], [2], [3]). Randomness may be in line with the major features of real-life networks, but as mentioned by Baraba'si et al., it makes difficult to gain a profound understanding of how networks are developed, and how do different vertices relate to each other (Ref. [4]). Therefore, it is not easy to described the topological characteristics, we decided to approximate the character of random networks by research the character of the minimal and maximal random network models, where the minimal model have minimal vertices and edges, similarly, the numbers of vertices and edges of maximal model are maximal respectively. This way provide a new method as we described the nature of the random networks.

Here, we construct a new network model by network operations, then explore the minimal and maximal models of which to research the nature of new random network models. Our idea for exploring operations of network models is motivated from the knowledge of graph theory (Ref. [5], [6], [7], [12]). We, by constructing network models, have shown: For any network $N_{1}, N_{2}, \ldots, N_{n}$ and one or more network operations, these networks do operations to each other, then, it will produce more and more new network models. The nature of the new model with the original models with the same or similar? The network operation with closeness to good properties for the networks whether or not? In this paper, we define two network operations and construct new network models on the basis of Apollonian network models.

Scale-free is important character of the networks, since scale-free networks possess better properties and constructions. It has been proven that many real networks are scale-free networks. In "Emerge of scaling in random networks", two physical scientists Baraba'si and Albert have shown the degree distribution follows a power-law $P(k) \sim k^{-\lambda}$ to be one of standard properties of a scale-free network $N(t)$, where $P(k)$ is the probability distribution of a vertex joined with $k$ vertices in $N(t)$, and $\lambda$ within the range $2<\lambda<3$. In this paper, we pay more attention to the scale-free of new $A S$-mixed network models, as well as other nature, such as edge distribution and velocity. 


\section{Random $A S$-mixed network models and their properties}

\subsection{New operations}

In this section we introduce two new operations before we show our models, the named as triangle-expanding operation and triangle-contracting operation. They are a pair of opposite operations.

1) Triangle-expanding operations: Let $u$ is a vertex having three neighbor vertices $A, B, C$, as well as $u$ and it's neighbor vertices are joined by an edge respectively (see Figure 1(a)). We call it a basic configuration can expand (BCCE), we expand $u$ into a triangle $a b c$, and furthermore join $a$ with $A$ and $B$ by two edges, join $b$ with $A$ and $C$ by two edges, and then join $c$ with $B$ and $C$ by two edges. We get another configuration (see Figure 1(b)), We called a basic configuration can contract $(B C C C)$. Clearly, after the graph $B C C E$ expand, the graph $B C C C$ more 2 vertices and 6 edges than graph $B C C E$. We defined a labelling function $f$ as: $f(A)=f(B)=f(C)=k-1, f(a)=f(b)=f(c)=f(u)=k$ for $k \geq 1$.

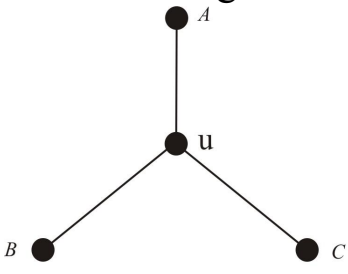

(a) $B C C E$

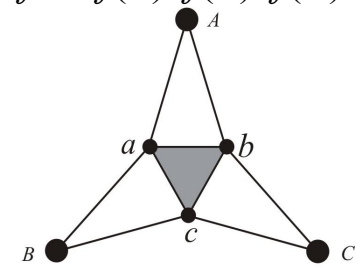

(b) $B C C C$

Figure.1 (a) BCCE is a basic configuration can expand, (b) BCCC is a basic configuration can contract.

2) Triangle-contracting operations: For graph $B C C C$, we contract triangle $a b c$ into a vertice $u$, and join $u$ with $A, B$ and $C$ respectively, merely, triangle-contracting operation is inverse operation of triangle-expanding operation(see Fig.2). We are ready for constructing the desired models.

\subsection{Building Random AS-mixed network models}

First of all, we introduce a fractal operation yields a configuration $H$ in the way: Add a vertex $v$ into the inner face of a triangle and then join $v$ with three vertices $A, B$ and $C$ by three edges, respectively. We define a labelling function $\mathrm{g}$ for $H(1)$ as: $g(\mathrm{~A})=g(\mathrm{~B})=g(\mathrm{C})=k-1, g(u)=k$ for $k \geq 1$.

2-dimension Apollonian model $\boldsymbol{A ( t )}$. The Apollonian model $A(\mathrm{t})$ (Ref. [8]) can be generated by the Apollonian-algorithm.

Apollonian-algorithm

Initialization. At time step $t=0, A(0)$ is a triangle $A B C$, and the labelling function $f$ holds $f(A)=f(B)=f(C)=0$.

Iteration. At time step $t$, do a fractal operation to every triangle $x y z$ of $A(t-1)$ with at least one of three labels $f(x), f(y)$ and $f(z)$ is equal to $t-1$, and label the vertex added with $t$ under $f$.

See $A(0), A(1), A(2)$ and $A(3)$ shown in Figure 3.

Next, we make a $A S$-mixed network model $A_{S}(t)$ by the triangle-expanding operations, and it can be constructed in an algorithm: For $t=0$, we let $A_{S}(0)$ be the initial network having three vertices and three edges such that any pair of vertices is joined by an edge formed a triangle. We defined a labeling $f$ such that $f(\alpha)=0$, for each vertex $\alpha \in V(0)$. $A S$-mixed network models structure before $t$ time step is same to Apollonian network models structure at time step $t, A S$-mixed network model have $3^{t^{-}}$new vertices added into the network, their labeling both $f(\beta)=t$ for each vertex $\beta \in V(t) \backslash V(0) \cup V(1) \cup \ldots \cup$ $V(t-1)$. We choose randomly $\left\lceil\mathrm{p} \cdot 3^{t^{-}}\right\rceil$new vertices do triangle-expanding operations, we get random $A S$-mixed network models, where $p$ is a adjustment coefficient and $0 \leq p<1$. We can see $A_{S}(2)$ shown in figure 4. 

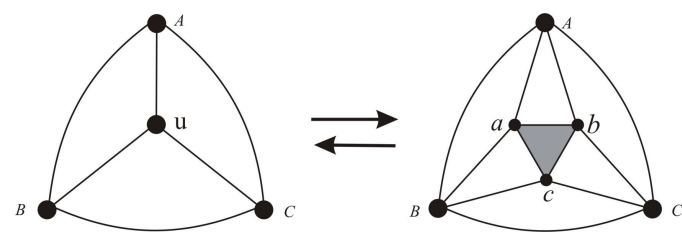

Figure 3. From left to right it is a triangle-expanding operation, from right to left it is a triangle-contracting operation.
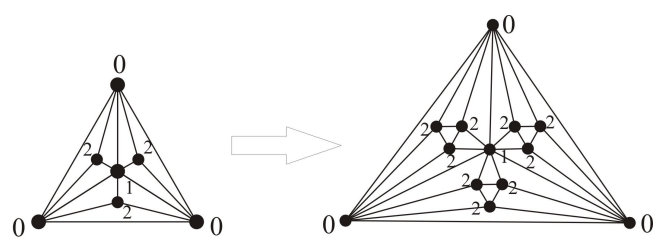

Figure 4. The construction of the AS-mixed network model at the time step 2 .

In order to further explore the nature of random $A S$-mixed network models, we can see the minimal model of it is Apollonian networks, because $p=0$ in this case, we denoted the model is $A S^{\min }(t)$ or directly $A(t)$, On the other hands, when $p=1$ we get the maximal model of random $A S$-mixed network models, denoted $A s^{\max }(t)$. Follows, we approximate the nature of new networks by research Apollonian networks $A(t)$ and $A_{S}{ }^{\max }(t)$ model. Let $n_{v}(t)$ and $n_{e}(t)$ be the total numbers of vertices and edges at time step $t$ respectively in $A_{S}(t)$, thereby, we have

$$
\begin{aligned}
& n_{v}^{\min }(t)=n_{v}^{4}(t)=\frac{3^{t}+5}{2}, n_{c}^{\min }(t)=n_{c}^{4}(t)=\frac{3^{t+1}+3}{2} \\
& n_{v}^{\max }(t)=\frac{3^{t}+5}{2}+2 \cdot 3^{t-1}=\frac{7 \cdot 3^{t-1}+5}{2} \\
& n_{c}^{\max }(t)=\frac{3^{t+1}+3}{2}+6 \cdot 3^{t-1}=\frac{21 \cdot 3^{t-1}+3}{2}
\end{aligned}
$$

We know $n_{v}^{A}(t)<n_{v}(t)<n_{v}^{\max }(t)$, similarly, we have $n_{e}^{A}(t)<n_{e}(t)<n_{e}^{\max }(t)$, So, we have the average vertex

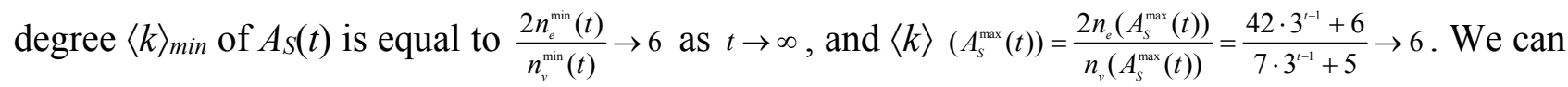
see when $t$ is large enough the resulting network is a sparse graph whose vertices have many fewer connections than is possible.

\subsection{Two types of degree cumulative distributions}

For estimating the topological structure of $A_{S}(t)$, we compute the min-cumulative and max-cumulative distributions $P_{\mathrm{ata}}^{\min }(k)$ and $P_{\mathrm{san}}^{\operatorname{mox}}(k)$, and the min-edge and max-edge cumulative distributions $P_{\text {exim }}^{\min }(k)$ and $P_{\text {ecei }}^{\max }(k)$ for two $A S$-mixed models $A(t)$ and $A S^{\max }(t)$, respectively, in the following argument. Since the degree spectrums of two $A S$-mixed models $A_{S}(t)$ are discrete, so we can use a technique introduced by Newman in ([9], [10]\}). Dorogovstev said the cumulative distribution

$$
P_{\text {cum }}(k)=\sum_{k^{\prime} \geq k} \frac{\left|V\left(k^{\prime}, t\right)\right|}{n_{v}(t)} \sim k^{1-\lambda}
$$

where $\left|V\left(k^{\prime}, t\right)\right|$ is the number of vertices of degree $k^{\prime}$ (Ref. [10]).

We can easily to gain the degree spectrum of the 2-dimension Apollonian networks

\begin{tabular}{|l|l|l|l|l|l|l|}
\hline$d$ & 3 & 6 & 12 & $\ldots$ & $3 \cdot 2^{t-1}$ & $2^{1+1}$ \\
\hline$n_{d}(t)$ & $3^{1-1}$ & $3^{t-2}$ & $3^{t-3}$ & $\ldots$ & 1 & 3 \\
\hline
\end{tabular}

and $A(t)$ do triangle-expanding operations for every vertex with labeling $f(u)=t$, we get the maximal model of AS-mixed network model, it's degree spectrum is following

\begin{tabular}{|l|l|l|l|l|l|l|}
\hline$d$ & 4 & 18 & 36 & $\ldots$ & $3 \cdot 2^{t-1} \cdot 3$ & $3 \cdot 2^{t-1}+1$ \\
\hline$n_{d}(t)$ & $3^{t}$ & $3^{t-2}$ & $3^{t-3}$ & $\ldots$ & 1 & 3 \\
\hline
\end{tabular}
$P_{\text {cim }}^{\min }(k)=\sum_{k 2 k} \frac{\left|V\left(k^{\prime}, t\right)\right|}{n_{v}(t)}=\frac{3+1+3+3^{2}+\cdots+3^{t-1}}{\frac{3^{t}+5}{2}} \sim 3^{t-1}$

where $\tau$ is the time that vertices with degree $k$ added in the $A S$-mixed networks, plugging $\tau=t-\frac{\ln k-\ln 3}{\ln 2}$ into the above equation, and when $t$ is large enough, we can obtain

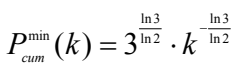


So, the min-cumulative distribution $P_{c \mathrm{~m}}^{\min }(k)$ follows a power law form with the exponent $\gamma=1+\frac{\ln 3}{\ln 2}$, and $2<\gamma<3$. It indicates that the minimal model of random $A S$-mixed network or called Apollonian network model are scale-free network.

For $A s^{\max }(t)$, we have the max-cumulative distribution

$P_{\text {cum }}^{\max }(k)=\sum_{k 2 k} \frac{\left|V\left(k^{\prime}, t\right)\right|}{n_{v}(t)}=\frac{3+1+3+3^{2}+\cdots+3^{k-2}+3^{x}}{\frac{7 \cdot 3^{t-1}+5}{2}} \sim 3^{r-t}$

plugging $\tau=t-\frac{\ln k-\ln 9}{\ln 2}$ into the above equation, and when $t$ is large enough, we can obtain $P_{\text {cum }}^{\max }(k)=3^{\frac{\ln 9}{\ln 2}} \cdot k^{\frac{\ln 3}{\ln 2}}$

Similarly, we can easily compute the $2<\gamma=1+\frac{\ln 3}{\ln 2}<3$. Figure5 indicates the degree cumulative distributions of Apollonian network and randomly maximal $A S$-mixed network, abscissa is refer to the degree of vertices, and ordinate is refer to the degree cumulative of network models. The solid line and dotted line describe the minimal model and the maximal model respectively. So we think the both of random $A S$-mixed network is also scale-free. Observe (6) and (8), we can see

$$
P_{c u m}^{\max }(k)=3^{\frac{\ln 3}{\ln 2}} P_{c u m}^{\min }(k)
$$

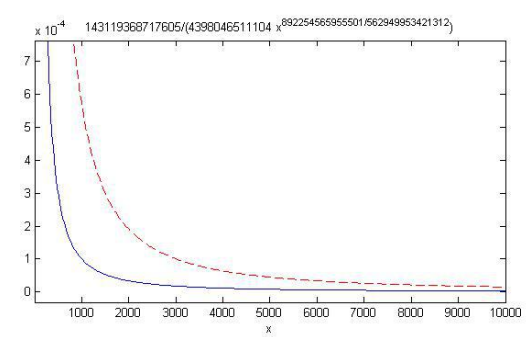

Figure 5. The solid line is degree cumulative distribution of Apollonian networks, the dotted line is degree cumulative distribution of $A S^{\max }(t)$.

\subsection{Two types of edge cumulative distributions}

Motivated from the cumulative degree distribution that is an important character of scale-free networks the authors in [3] proposed a statistic way named as $P_{\text {ecum }}(k)=\frac{1}{n_{e}(t)} \sum_{i=0}^{\tau} n_{e}(i)$ for $0<\tau<t$. For Apollonian networks, we compute the min-edge cumulative distribution is

$P_{\text {ecum }}^{\min }(k)=\frac{1}{n_{e}^{\text {min }}(i)} \sum_{k \gtrless k} k^{\prime}\left|V\left(k^{\prime}, t\right)\right|=\frac{3\left(2^{z}+1\right)+\sum_{i=1}^{z} 3^{i} 2^{r-i}}{n_{e}^{\min }(i)} \sim C_{1} 3^{\tau-t}$

where $n_{e}^{\min }(i)=\frac{3^{t+1}+3}{2}$, and $C_{1}$ is a constant. On the other hand, we get another max-edge cumulative distribution as follows

$$
P_{e c u m}^{\max }(k)=\frac{1}{n_{e}^{\max }(i)} \sum_{k \geq k} k^{\prime} \cdot\left|V\left(k^{\prime}, t\right)\right|=\frac{3\left(2^{\tau}+2^{r-1}+1\right)}{n_{e}^{\max }(i)}+\frac{9}{n_{e}^{\max }(i)} \sum_{i=0}^{r-1-1}\left(3^{\prime} 2^{r-i-1}+4 \cdot 3^{\tau}\right) \sim C_{2} 3^{r-1}
$$

where for $n_{e}^{\max }(i)=\frac{21 \cdot 3^{t-1}+3}{2}$, and $C_{2}$ is also a constant. It is clear that two edge cumulative distributions are equivalent to two cumulative distributions, respectively. Thereby, we conclude that two edge cumulative distributions obey power-laws.

\subsection{Velocities of the models}

The dynamic development speed of networks was proposed by (Ref. [11]), this metric is used to measure the speed development of real networks. The velocity of the minimum random $A S$-mixed network $A(t)$ is

$$
V_{e l}(A(t))=\frac{\partial n_{v}(A(t))}{\partial t} \cdot \frac{\partial n_{c}(A(t))}{\partial t}=\frac{1}{2} 3^{t} \ln 3 \cdot \frac{3}{2} 3^{t} \ln 3=\frac{3}{4}\left(3^{t} \ln 3\right)^{2}
$$


For the randomly maximal $A S$-mixed network $A S^{\max }(t)$, it's velocity is

$V_{e l}\left(A_{s}^{\max }(t)\right)=\frac{\partial n_{v}\left(A_{s}^{\max }(t)\right)}{\partial t} \cdot \frac{\partial n_{e}\left(A_{s}^{\max }(t)\right)}{\partial t}=\frac{7}{6} 3^{t} \ln 3 \cdot \frac{21}{6} 3^{t} \ln 3=\frac{49}{12}\left(3^{\prime} \ln 3\right)^{2}$

It is easy to know that $V_{e l}(A(t))<V_{e l}(t)<V_{e l}\left(A_{s}^{\max }(t)\right)$ where $V_{e l}(t)$ is the velocity of $A_{S}(t)$. Furthermore, $\frac{V_{e l}(A(t))}{V_{e l}\left(A_{s}^{\max }(t)\right)}=\frac{9}{49} \sim \frac{1}{5} \Rightarrow 5 V_{c l}(A(t)) \approx V_{c l}\left(A_{s}^{\max }(t)\right)$ We can control $V_{e l}(t)$ by computing $V_{e l}(A(t))$ and $V_{c l}\left(A_{s}^{\max }(t)\right)$. It can simulate the real-network because of its randomness, this $A S$-mixed network can satisfy higher requirements on the speed of network, such as we can curb the speed of rumors and prevent the speed of infectious diseases by constructing new models.

\section{Conclusions}

In this paper, we are aiming construction of quality network models $A_{S}(t)$, due to the mix process of model is random, so we regard $A_{S}(t)$ is a partial random $A S$-mixed model. We also defining two new network operations, our operations are dynamic according to time and are not stable like that in graph theory. As known the Apollonian networks $A(t)$ are scale-free, it returned $A_{S}(t)$ by doing operations, $A_{s}(t)$ are also scale-free networks. So we constructing network models by preserving good network properties, we guess the operations of network can retain scale-free, but also the high clustering and small-world effect and so on.

People want to accurately depict random network topological characteristics is very difficult, so our idea is to use the upper and lower bounds to compress the random network, and the difference between the upper and lower bounds is very small, which indicates that our assertion is correct. This way provide a new method as we described the nature of the random networks and it can help us to explore more and more real random networks, hence, we can get the rule of random networks, which it is unknown before.

\section{Acknowledgment}

The authors are grateful to the anonymous referees for their valuable comments and suggestions. The third author, Bing Yao, thanks the National Natural Science Foundation of China under grants No. 61163054, No. 61363060 and No. 61662066. The fourth author, Ming Yao, thanks The Special Funds of Finance Department of Gansu Province of China under grant No. 2014-63.

\section{References}

[1] R.Albert and A.-L.Barabasi, Rev.Mod.Phys.74,47(2002)..

[2] M.E.J.Newman, SIAM Review 45,167(2003).

[3] S.N.Dorogovtsev and J.E.F.Mendes, Evolution of Networks: From Biological Nets to the Internet and WWW(Oxford University Press, New York, 2003).

[4] A.-L.Barabasi, E.Ravasz, and T.Vicsek, Physica A 299,559(2001).

[5] Bing Yao, Chao Yang, Ming Yao, Hongyu Wang, Xiang'en Chen, Xiaomin Zhang, Mogang Li. Graphs As Models of Scale-free Networks. Applied Mechanics and Materials, Vol.380-384(2013) pp 2034-2037. DOI: 10.4028/www.scientific.net/AMM.380-384.2034

[6] Bing Yao, Hongyu Wang, Ming Yao, Xiang'en Chen, Chao Yang and Xiaomin Zhang. On The Collapse of Graphs Related to Scale-Free Networks. Proceeding of ICIST2013. Third International Conference on Information Science and Technology March 23-25,2013,738-743.

[7] Bing Yao, Xia Liu, Wanjia Zhang, Xiang'en Chen, Xiaomin Zhang, Ming Yao, Zheng-Xue Zhao. Applying Graph Theory To The Internet Computing and Communications and 2013 IEEE International Conference in Embedded and Ubiquitous Computing, 2354-2361. DOI:10.1109/HPCC.and.EUC.2013.339. 
[8] Zhongzhi Zhang, Francesc Comellas, Guillaume Fertin and Lili Rong. High dimensional Apollonian networks. E-print cond-mat/0503316.

[9] M.E.J.Newman, The structure and function of complex networks, SIAM Review 45(2003) $167-256$.

[10] S.N.Dorogovstev, A.V. Goltsev, J.F.F. Mendes. Pseufractal scale-free web. Physiacal review 2002, 65, 066122-066125.

[11] Bing Yao, Xiaomin Wang, Jing Su, Fei Ma, Ming Yao, Mingjun Zhang, and Jianmin Xie. Methods And Problems Attempt in Scale-Free Models From Complex Networks. Submitted.

[12] Bing Yao, Chao Yang, Ming Yao, Hongyu Wang, Xiang'en Chen, Xiaomin Zhang, Mogang Li. Applied Mechanics and Materials, Vol. 380-384(2013) pp 2034-2037. Bing Yao, Hongyu Wang, Ming Yao, Xiang'en Chen, Chao Yang and Xiaomin Zhang. Third International Conference on Information Science and Technology March 23-25, 2013, 738-743. Bing Yao, Xia Liu, Wan-jia Zhang, Xiang'en Chen, Xiao-min Zhang, Ming Yao, Zheng-xue Zhao. 2013 IEEE International Conference on High Performance Computing and Communications and Embedded and Ubiquitous Computing, 2354-2361. Bing Yao, Xia Liu, Wanjia Zhang, Xiang'en Chen, Ming Yao. Applied Mechanics and Materials, Volumes 513-517, pp2444-2448. Bing Yao, Xia Liu, Wanjia Zhang, Xiang'en Chen, Ming Yao. Proceeding of 2014 IEEE 7th Joint International Information Technology and Artificial Intelligence Conference (ITAIC 2014), pp 450-454. 